\title{
LA-UR-21-23887
}

Approved for public release; distribution is unlimited.

Title: $\quad \operatorname{MCNP}(\mathrm{R})$ Particle Transport Code: Its History and Future

Author(s): $\quad$ Rising, Michael Evan

Intended for: $\quad$ Report

Issued: 
Disclaimer:

Los Alamos National Laboratory, an affirmative action/equal opportunity employer, is operated by Triad National Security, LLC for the National Nuclear Security Administration of U.S. Department of Energy under contract 89233218CNA000001. By approving this article, the publisher recognizes that the U.S. Government retains nonexclusive, royalty-free license to publish or reproduce the published form of this contribution, or to allow others to do so, for U.S. Government purposes. Los Alamos National Laboratory requests that the publisher identify this article as work performed under the auspices of the U.S. Department of Energy. Los Alamos National Laboratory strongly supports academic freedom and a researcher's right to publish; as an institution, however, the Laboratory does not endorse the viewpoint of a publication or guarantee its technical correctness. 


\title{
MCNP ${ }^{\circledR}$ Particle Transport Code
}

\section{Its History and Future}

\author{
Michael E. Rising \\ MCNP Technical Lead \\ XCP-3: Monte Carlo Group
}

April 2021 


\section{Introduction to MCNP}




\section{Legal Notice}

MCNP ${ }^{\circledR}$ and Monte Carlo N-Particle ${ }^{\circledR}$ are registered trademarks owned by Triad National Security, LLC, manager and operator of Los Alamos National Laboratory for the U.S. Department of Energy under contract number $89233218 C N A 000001$. Any third party use of such registered marks should be properly attributed to Triad National Security, LLC, including the use of the $₫$ designation as appropriate. Any questions regarding licensing, proper use, and/or proper attribution of Triad National Security, LLC marks should be directed to trademarks@lanl.gov. For the purposes of visual clarity, the registered trademark symbol is assumed for all references to MCNP within the remainder of this report.
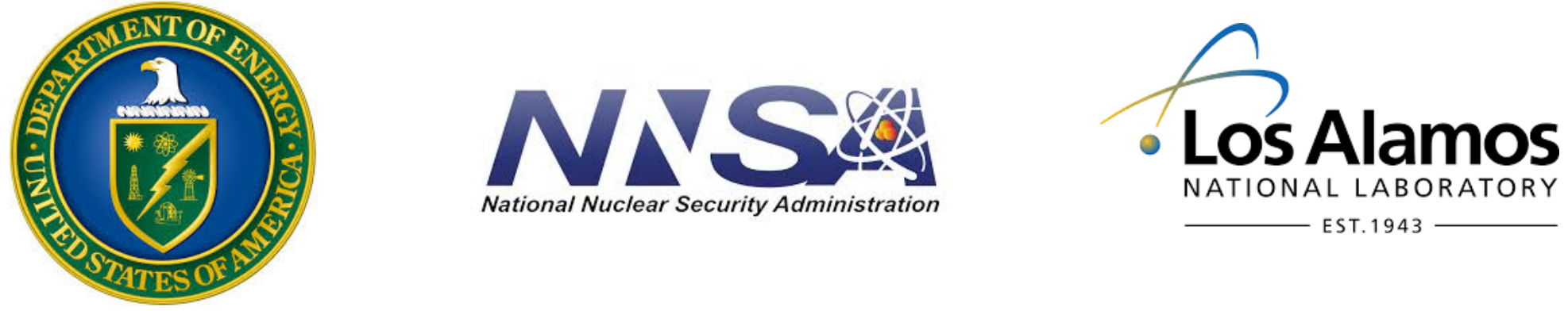


\section{Monte Carlo Particle Transport Codes Group}
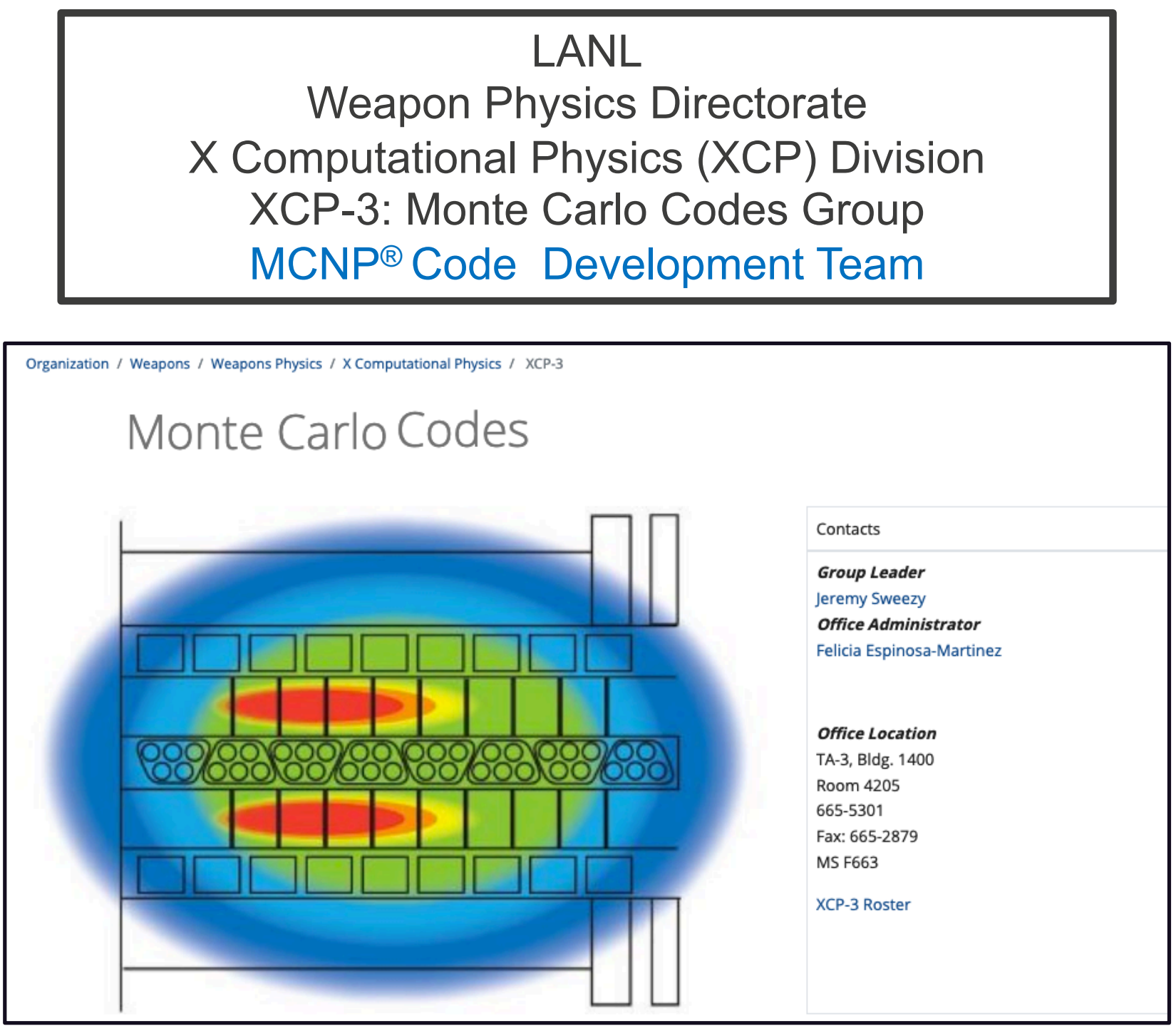


\section{Monte Carlo Particle Transport Method}

\section{An approach for simulating random walk of particles through matter on a computer.}

"Following each of a large number of particles from the source throughout its life history to its death ... using the elementary probabilities at each stage of its career in determining its fate" 1

(1) Geometry

Ray-tracing through "exact" model of problem geometry to determine locations of interactions.

(2) Physics

Random sampling using cross-section data, physics models, and source descriptions to simulate interactions.

(3) Tallies

Bookkeeping to record how often certain events occur during the simulation.
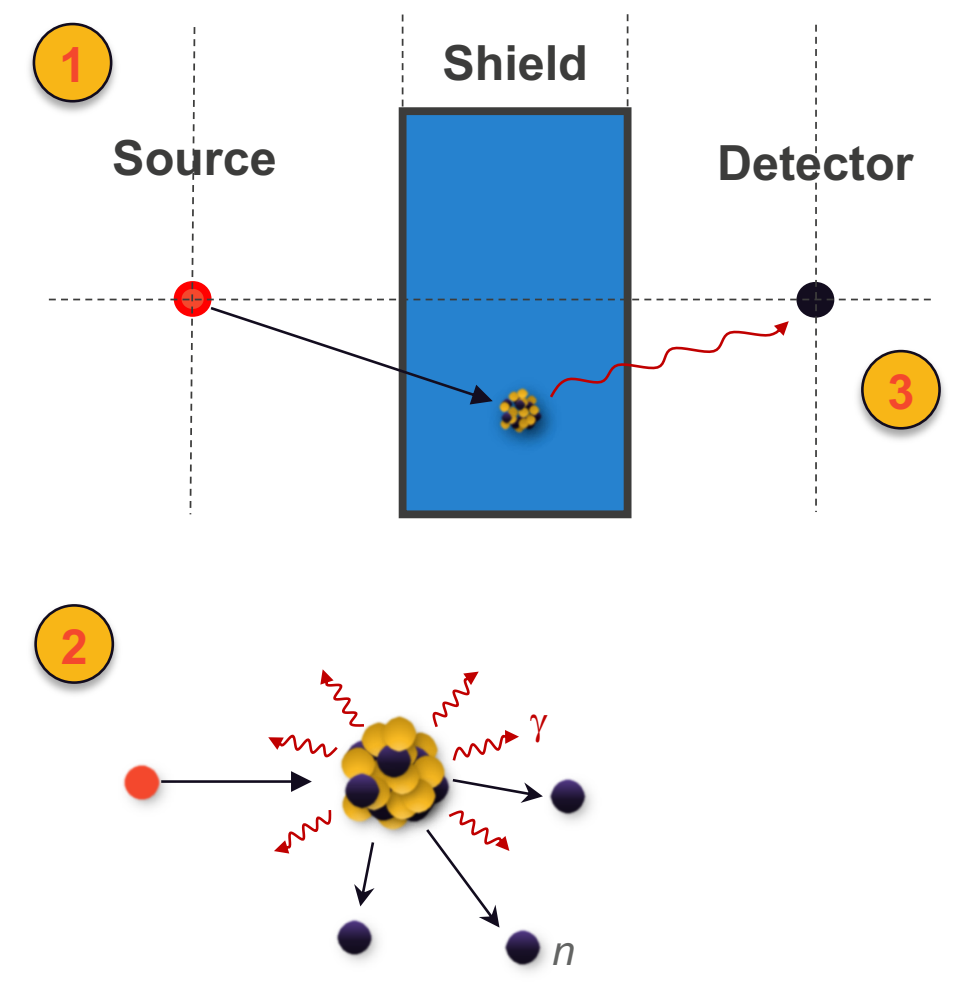


\section{MCNP is a Trusted Capability Used in the Nuclear Criticality and Reactor Physics Communities}

- For nuclear criticality, MCNP use is extensive and routine

- World class V\&V for criticality safety applications

- For both static critical and subcritical systems at NCERC

- MCNP is used extensively for criticality safety analysis throughout the DOE including PF-4 pit production applications

- For advanced reactor physics, MCNP use is growing

- Primarily used in static analysis, although multi-physics applications are growing

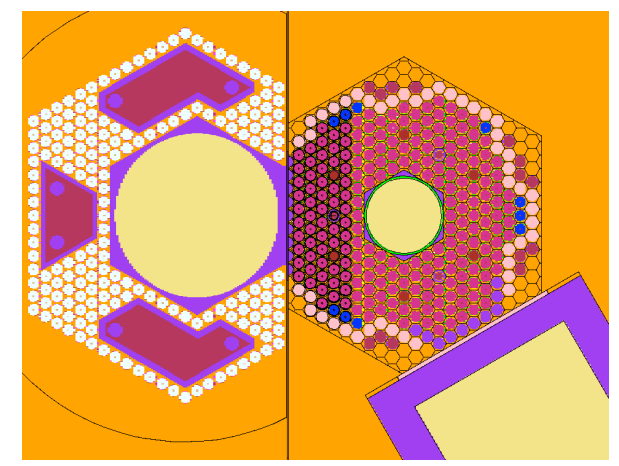

Sandia National Laboratory ACRR Reactor

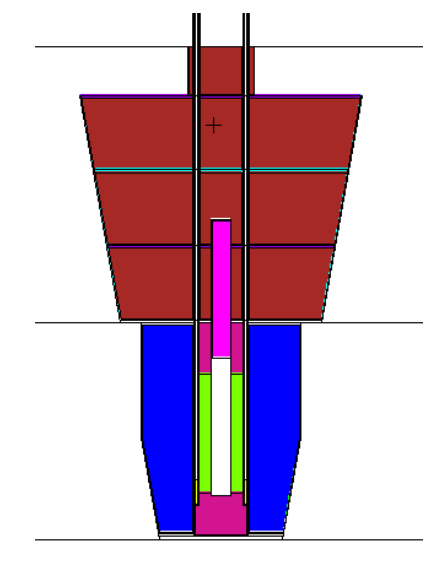

KRUSTY Experiment

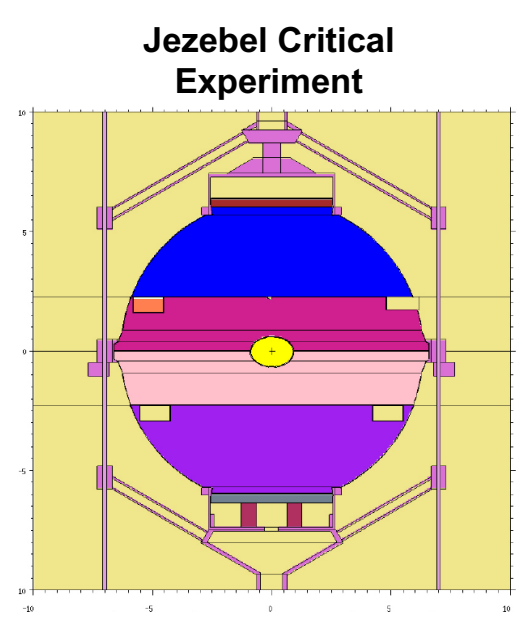

Subcritical BeRP Ball Experiment

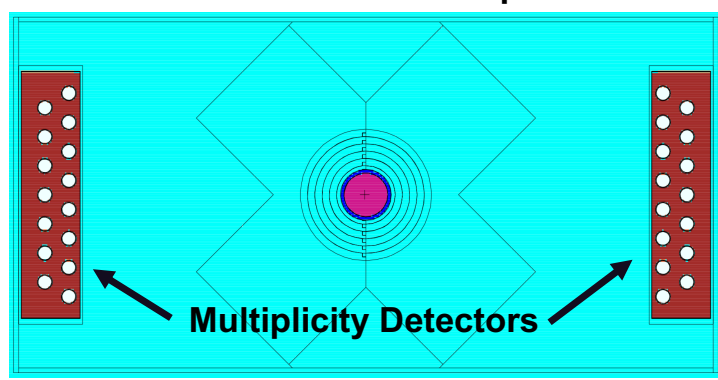




\section{MCNP Unstructured Mesh Applications are Growing - Active Feature Developments and Opportunities}

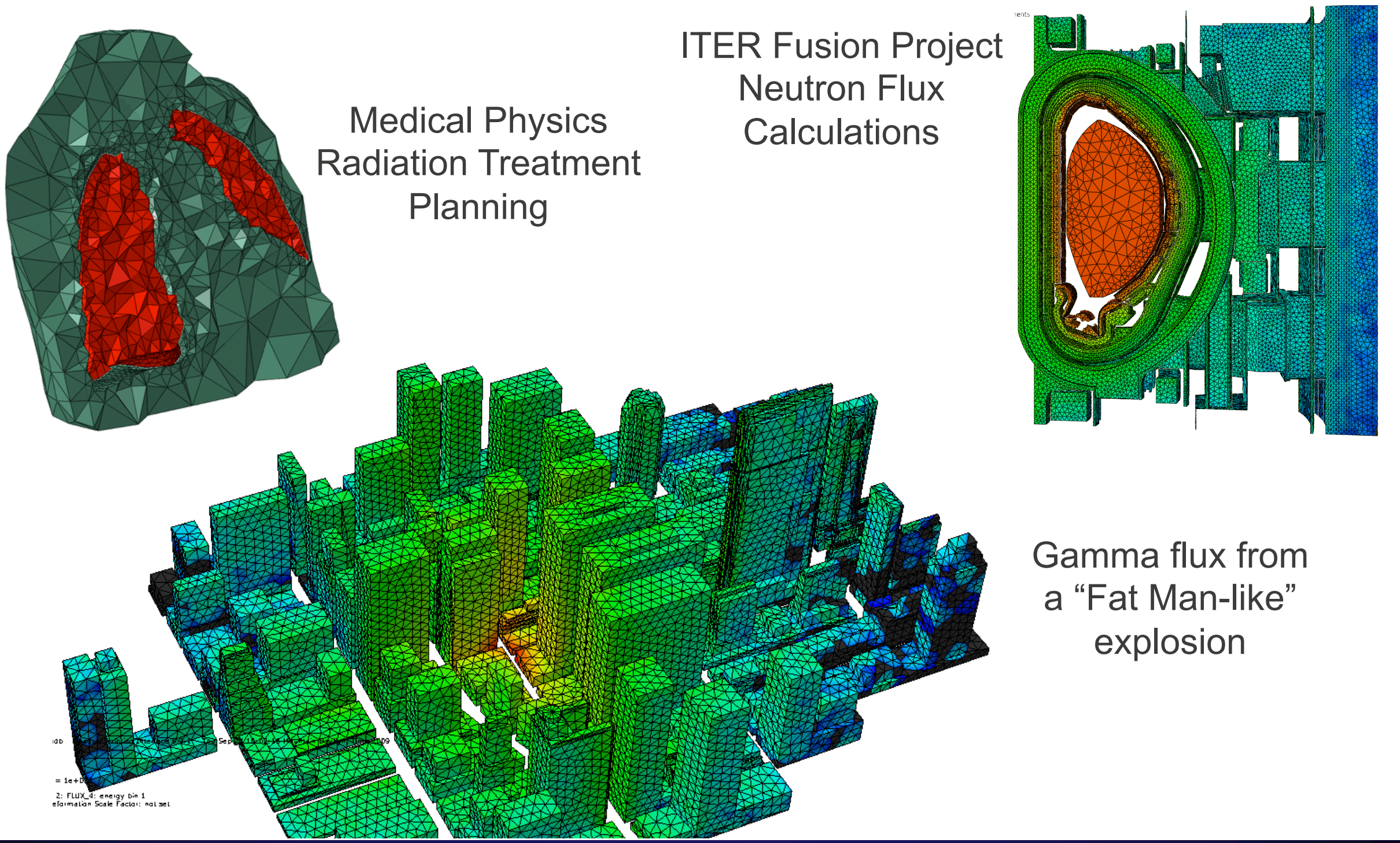




\section{History of MCNP}




\section{Creators of Monte Carlo Method}

- The emergence of the Monte Carlo method as a research tool springs from work done at Los Alamos in the 1940s.

- Creators: Stan Ulam, John von Neumann, Nicholas Metropolis, Robert Richtmyer, and Enrico Fermi.

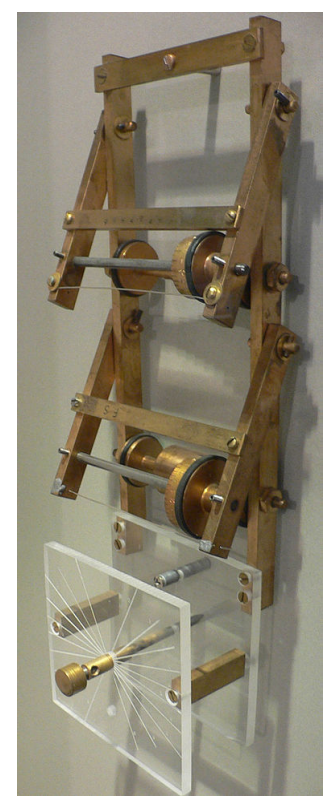

FERMIAC

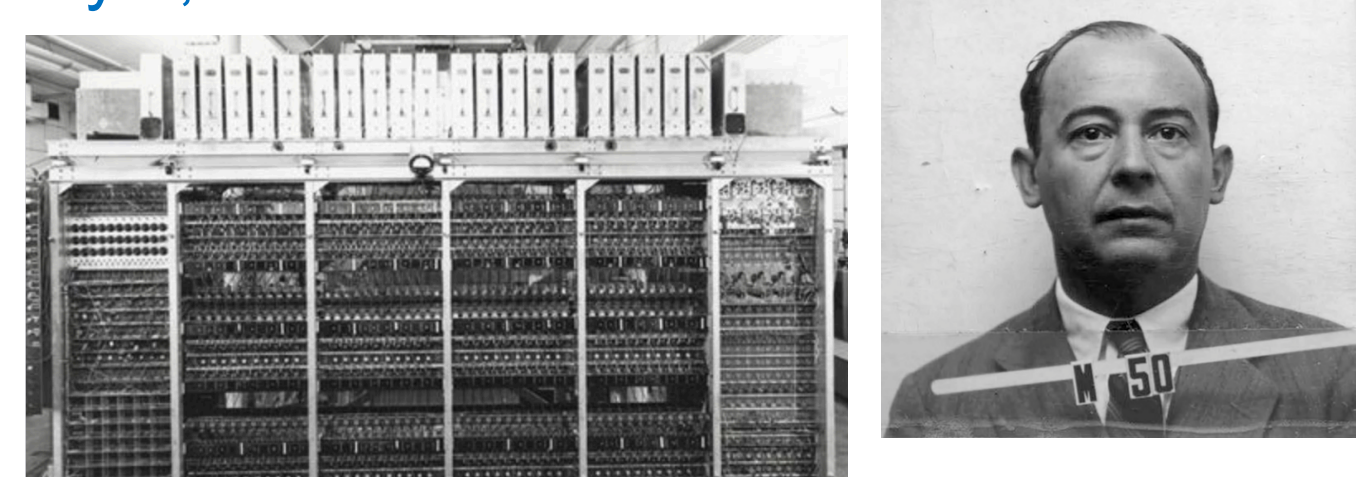

John von Neumann

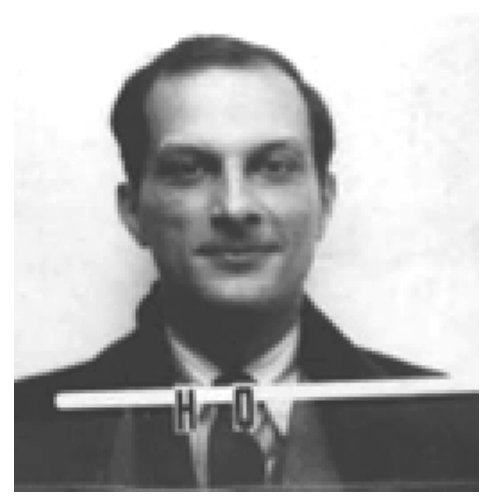

Stan Ulam

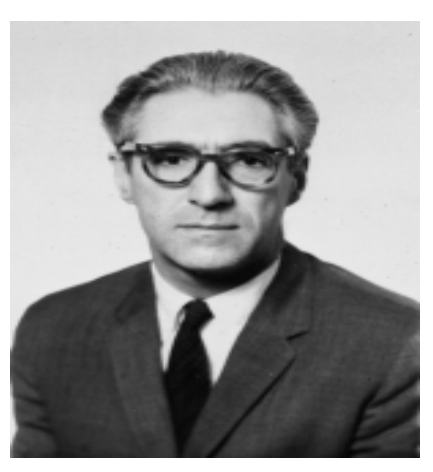

Nicholas Metropolis 


\section{The Origins of the Monte Carlo Method}

- In 1935, John von Neuman invited Stan Ulam to visit Institute for Advanced Study in Princeton.

- In 1943, von Neumann visited Los Alamos as a consultant.

- Ulam arrived in Los Alamos to join the Manhattan project on Feb 4, 1944.

- S. M. Ulam and J. von Neumann, Random ergodic theorems, Bull. Amer. Math, soc. vol. 51 (1945) p. 660.

- S. M. Ulam and J. von Neumann, On combination of stochastic and deterministic processes, Bull. Amer. Math. Soc. vol. 53 (1947) p. 1120.
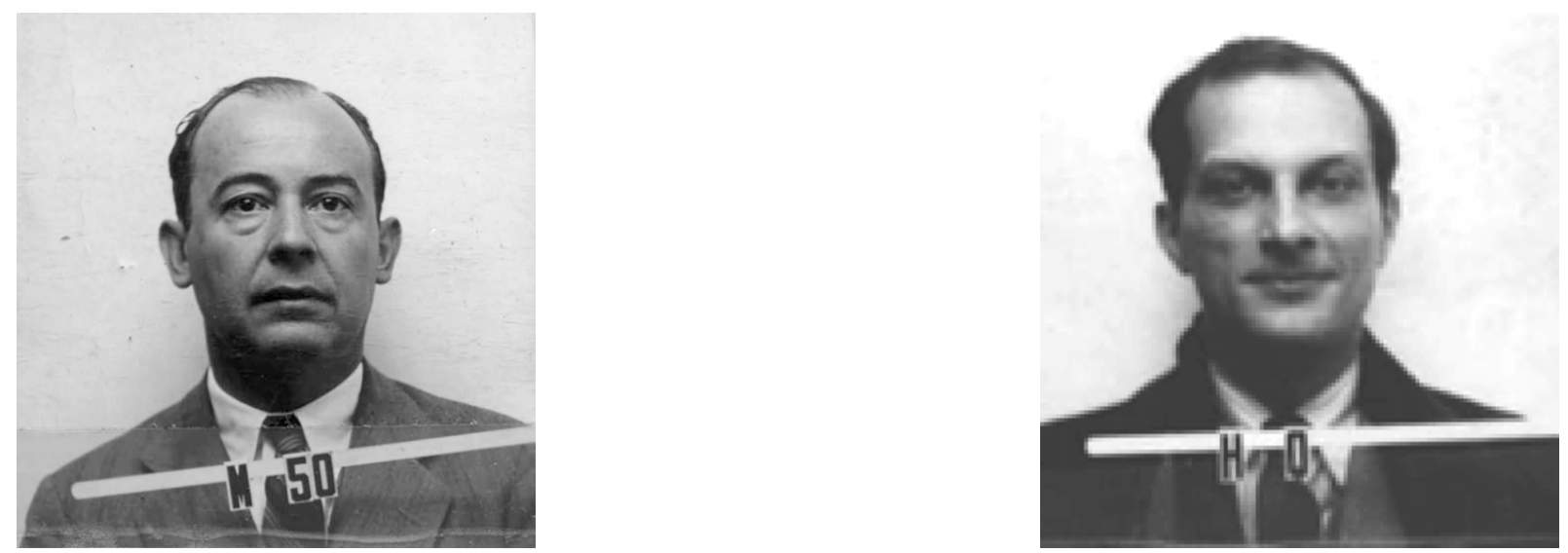


\section{The Origins of the Monte Carlo Method}

"I remember quite well how, very early in the Los Alamos Project, it become obvious that analytical work alone was often not sufficient to provide even qualitative answers. The numerical work by hand and even the use of desk computing machines would require a prohibitively long time for these problems. This situation seemed to provide the final spur for von Neumann to engage himself energetically in the work on methods of computation utilizing the electronic machines. ... consider the problem of diffusion in a region of space bounded by surfaces which partly reflect and partly absorb the diffusing particles. If the geometry of the region is complicated, it might be more economical to try to perform physically a large number of such random walks rather than to try to solve the integro-differential equations classically. These walks can be performed conveniently on machines and such a procedure in fact reverses the treatment which in probability theory reduces the study of random walks to the study of differential equations." 1 


\section{The Origins of the Monte Carlo Method}

403. S. M. Ulam and John von Neumann: On combination of stochastic and deterministic processes. Preliminary report.

A computational procedure for the study of various differential equationsordinary or partial -is investigated. It consists of a statistical model of the corresponding physical problem and involves a process which is a combination of deterministic and stochastic processes (see Bull. Amer. Math. Soc. Abstract 51-9-165). This procedure is analogous to the playing of a series of "solitaire" card games and is performed on a computing machine. It requires, among others, the use of "random" numbers with a given distribution. Various distributions of such numbers can, however, be obtained by deterministic processes. For example, starting with almost every $x_{1}$ (in the sense of Lebesgue measure) and iterating the function $f(x)=4 x \cdot(1-x)$ one obtains a sequence of numbers on $(0,1)$ with a computable algebraic distribution. By playing suitable games with numbers "drawn" in this fashion, one can obtain various other distributions, either given explicitly or satisfying given differential or integral equations. (Received September 3, 1947.) 


\section{The Origins of the Monte Carlo Method}

DeAr BOB,

This is the letter I promised you in the course of our telephone conversation on Friday, March 7th.

I have been thinking a good deal about the possibility of using statistical methods to solve neutron diffusion and multiplication problems, in accordance with the principle suggested by Stan Ulam. The more I think about this, the more I become convinced that the idea has great merit. My present conclusions and expectations can be summarized as follows:

(1) The statistical approach is very well suited to a digital treatment. I worked out the details of a criticality discussion under the following conditions:

(a) Spherically symmetric geometry.

(b) Variable (if desired, continuously variable) composition along the radius, of active material ( 25 or 49 ), tamper material ( 28 or Be or WC), and slowerdown material ( $\mathrm{H}$ in some form).

apxis 9.1947

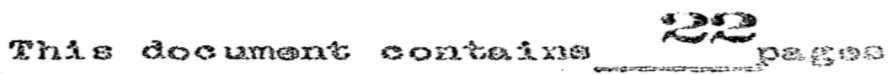




\section{The Origins of the Monte Carlo Method}

- "An incoming particle with great energy entering the atmosphere starts a whole chain of nuclear events. New particles are produced from the target nuclei, these in turn produce new reactions. This cascade process continues with more and more particle created until the available individual energies become too small to produce further nuclear events. The particles in question are protons, neutrons, electrons, gamma rays, and mesons. ..." - LADC-651, 1949

- "a medium in which a nuclear particle is introduced, capable of producing other nuclear particles with a distribution of energy and direction of motion. Assume here for simplicity that all particles are the same nature. Their procreative powers depend however, among others, on their position in the medium and on their energy. ..." - LADC-651

- "The statistical method can be applied by many computers in parallel and independently." - LADC-651 


\section{FERMIAC, ENIAC \& MANIAC}

- Monte Carlo Method for Radiation Transport Originated at LANL - Stanislaw Ulam, John von Neumann, Robert Richtmyer, and Nicholas Metropolis

- Early calculations performed on the FERMIAC11 and MANIAC machines

- Monte Carlo code development and applications have been an important part of LANL efforts since that time

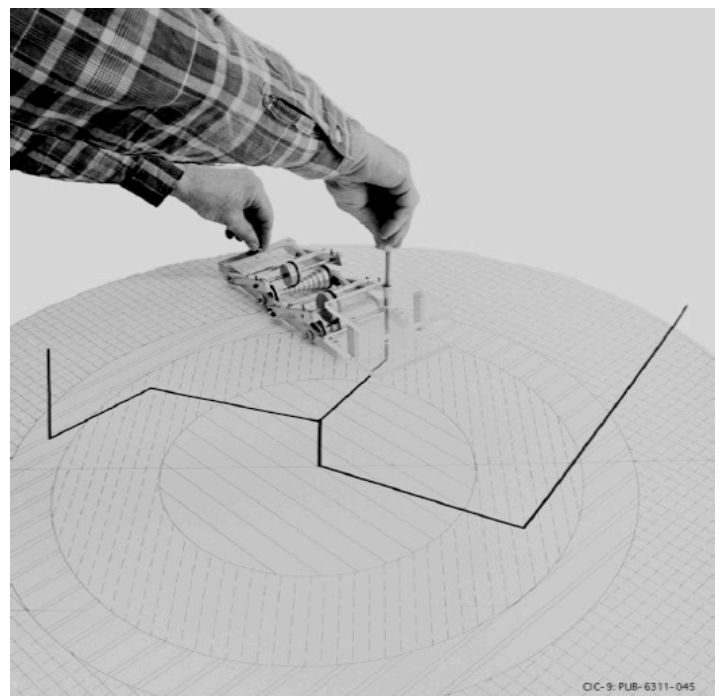

FERMIAC11 mechanically traced neutron paths

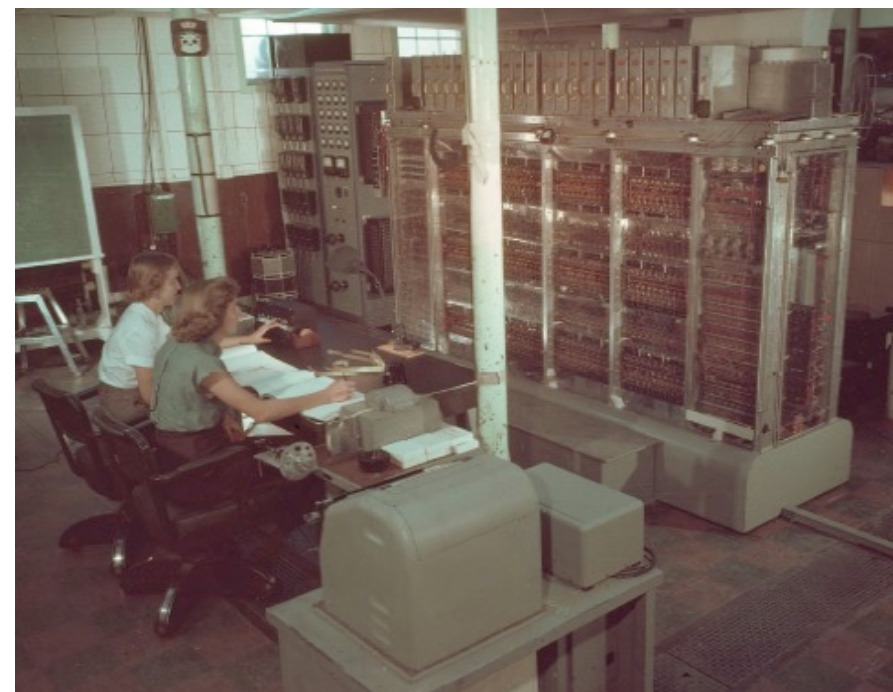

MANIAC computer performed early Monte Carlo calculations 


\section{FERMIAC, ENIAC \& MANIAC}

- Electrical Numerical Integrator And Calculator was the first generalpurpose electronic computer. (1946)

- MANIAC: Mathematical Analyzer, Numerical Integrator, And Computer

- MANIAC I 1952-1957

- MANIAC II 1957-1977
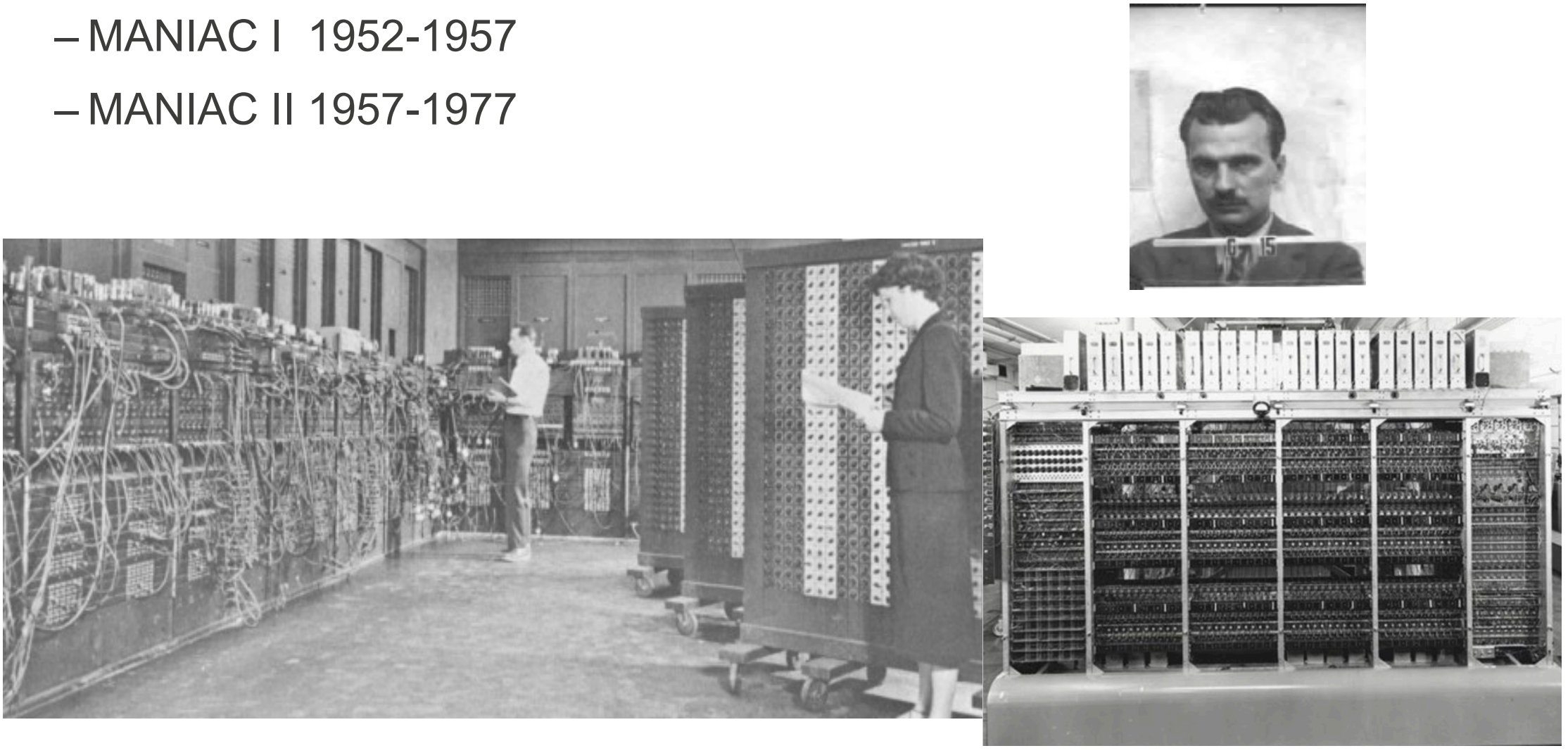


\section{MCNP ${ }^{\circledR}$ Version $1-06 / 21 / 1977$}

\section{MCNP: MCNG + MCP}

\section{LA.7396-M}

Manual

Special Distribution

|ssued: July 1978

\section{MCNP-A General Monite Carlo Code for Neutron and Photon Transport}

LASL Group TD.6
MCNP - A General Monte Carlo Code

for Neutron and Photon Transport

$$
\text { LASL Group TD-6 }
$$

\section{ABSTRACT}

The general purpose Monte Carlo code MCNP can be used for neutron, photon, or coupled neutron-photon transport. The code treats an arbitrary three-dimensional configuration of materials in geometric cells bounded by first- and second-degree surfaces and some special fourth-degree surfaces (elliptical tori).

Pointwise cross-section data are used. For neutrons, all reactions given in a particular cross-section evaluation (such as ENDF/B-IV) are accounted for. For photons, the code takes account of incoherent and coherent scattering, the possibility of fluorescent emission following photoelectric absorption, and absorption in pair production with local emission of annihilation radiation.

Standard optional variance reduction schemes include geometry splitting and Russian roulette, the exponential transformation, energy splitting, forced collisions in designated cells, flux estimates at point detectors, track-length estimators, and source biasing. 


\section{MCNP ${ }^{\circledR}$ Version $2-09 / 26 / 1979$}

\section{LA-7396-M, Revised}

Manual $1 \cdots 2$

\section{MCNP-A General Monte Carlo Code for Neutron and Photon Transport}

This manual is written as a practical guide for the use of our general-purpose Monte Carlo code MCNP. The intent is that the second chapter describe the mathematics, physics, and Monte Carlo simulation found in MCNP. However, this discussion is not meant to be exhaustive - details of the particular techniques and of the Monte Carlo method itself will have to be found elsewhere. The third chapter shows the user how to prepare input for the code. The fourth chapter contains several examples, and finally the fifth chapter explains the output. The appendices show how to use MCNP on a particular computer system at the Los Alamos Scientific Laboratory and also give details about some of the code internals that those who wish to modify the code may find useful.

Neither the code nor the manual is static. The code is changed from time to time as the need arises (about once a year), and the manual is changed to reflect the latest version of the code. This particular manual refers to Version 2 of MCNP that was released on September 26, 1979.

MCNP and this manual are the product of the combined effort of the people in Group X-6 of the Theoretical Applications Division (X Division) at the Los Alamos Scientific Laboratory. 


\section{MCNP (Monte Carlo N-Particle) Code}

- MCNP code is the world renowned Monte Carlo particle transport code developed at Los Alamos National Laboratory.

- MCNP is export controlled.

- MCNP external homepage: https://mcnp.lanl.gov

- MCNP internal homepage: https://xcp-confluence.lanl.gov/display/MCPUB

- MCNP version 1 was released in June 1977 [LA-7396-M]. - It was created by combing MCNG and MCP codes.

- MCNP version 3 was released for public distribution to Radiation Safety Information Computational Center (RSICC) in 1983.
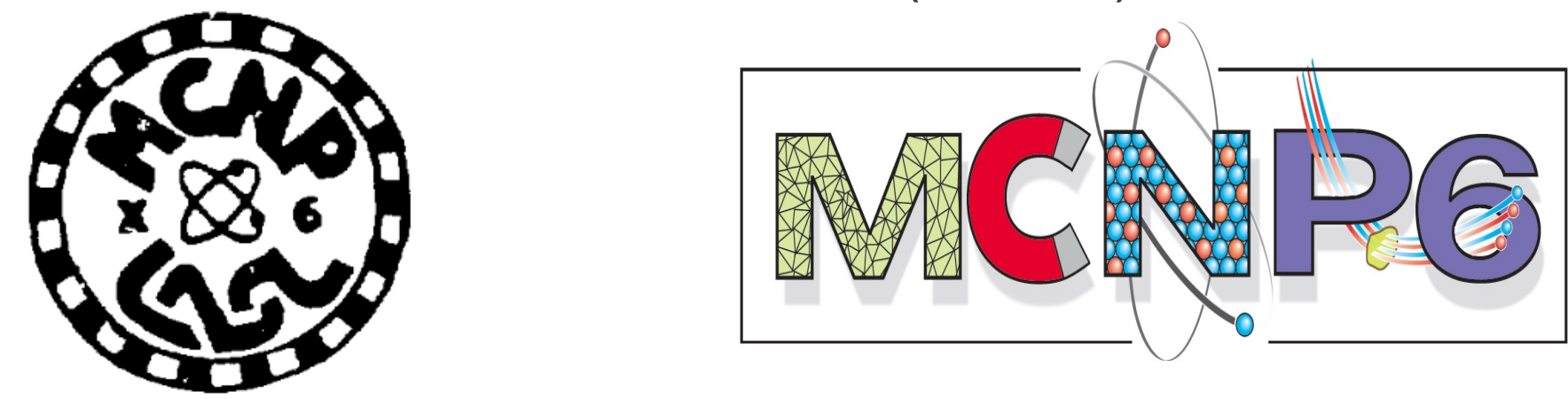


\section{MCNP Today}




\section{MCNP Version 6}

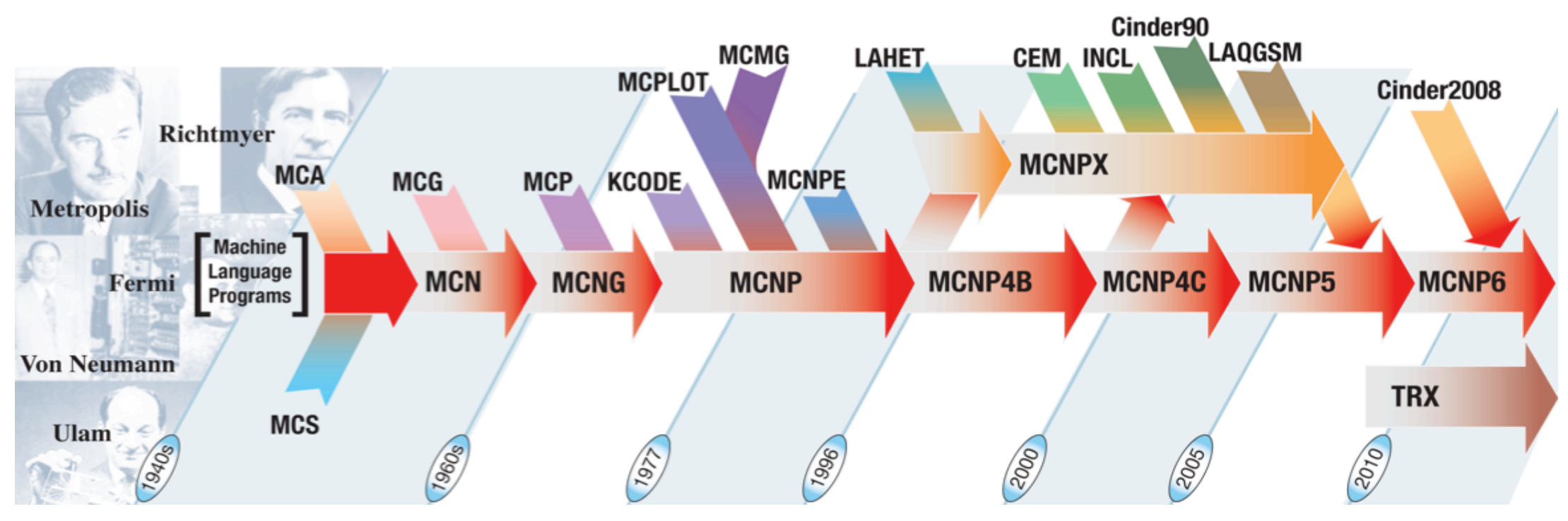

- MCNP 6.1 released by RSICC in July 2013

- MCNP 6.1.1 Update in July 2014

- MCNP 6.2 released to RSICC in April 2018

- MCNP 6.3 planned for release in 2021

- MCNP5 \& MCNPX are frozen

- no future releases

\begin{tabular}{|l|l|}
\hline \multicolumn{2}{|c|}{ FY19 MCNP } \\
Distributions \& Usage \\
\begin{tabular}{|l|l|}
\hline Distributor & Users \\
\hline LANL & $\sim 100$ \\
RSICC & $\sim 1,250$ \\
\hline & $\sim 50 \%$ of all RSICC \\
distributions
\end{tabular} \\
\hline
\end{tabular}




\section{From MCNP5 \& MCNPX to MCNP6}

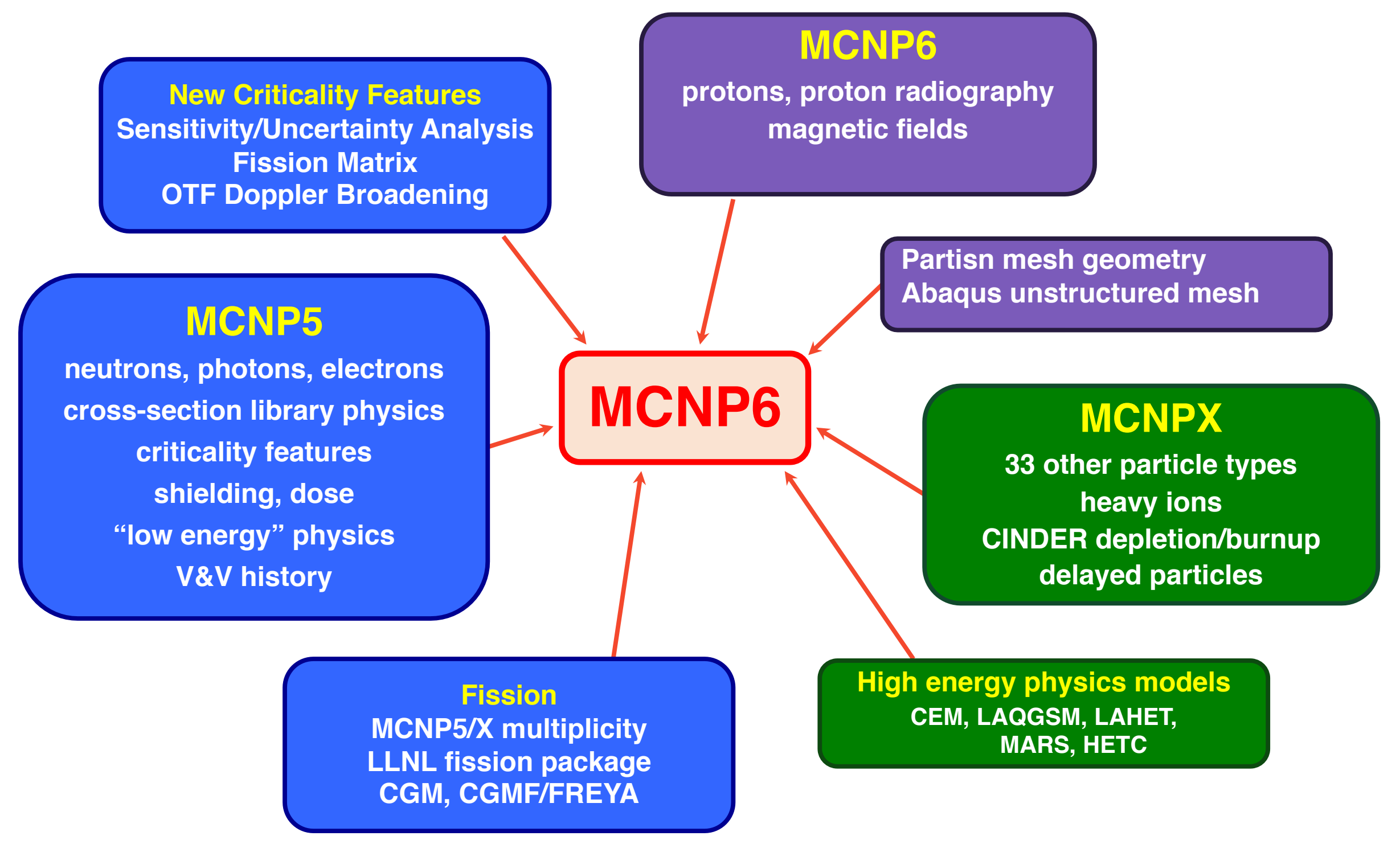




\section{Many LANL Core Missions and Global Applications Depend on the Capabilities MCNP Provides}

- MCNP provides needed capabilities for many important LANL applications areas

- Nuclear reactor physics

- Nuclear critical / subcritical experiments (NCERC)

- Criticality safety / operations (pit production)

- Nuclear diagnostics, survivability

- Nuclear weapon effects and outputs

- Emergency response / nuclear threat assessments

- Intrinsic radiation

- Nuclear safeguards and nonproliferation

- Radiation detection simulations (LANSCE)

Unstructured mesh city model used to study nuclear weapon effects

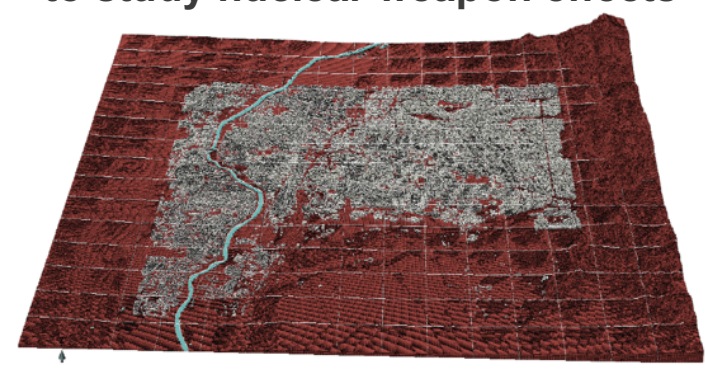

LANSCE cross section measurements enhanced by MCNP

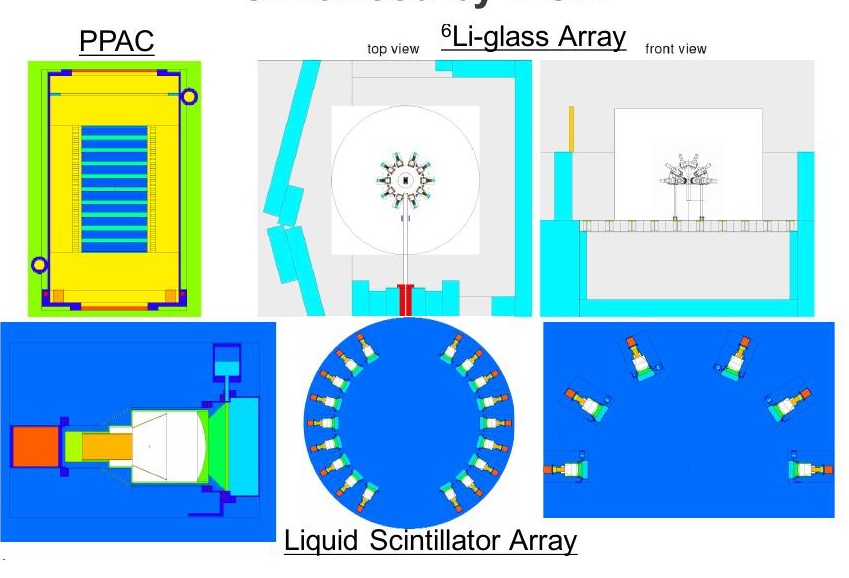

- Radiography (DARHT, pRad, NDSE, ECSE/Scorpius) 


\section{MCNP is the verified and validated transport code}

\section{which vets national nuclear data}

- MCNP has a long-standing reputation as the "goldstandard" Monte Carlo radiation transport code

- Plays a role in supporting the evaluations and interpreting experiments

- Nuclear data testing and validation relies heavily upon MCNP simulations

- $k$-eigenvalue, delayed neutrons, critical masses, reaction rate ratios, subcritical multiplication

- Quasi-differential, pulsed spheres
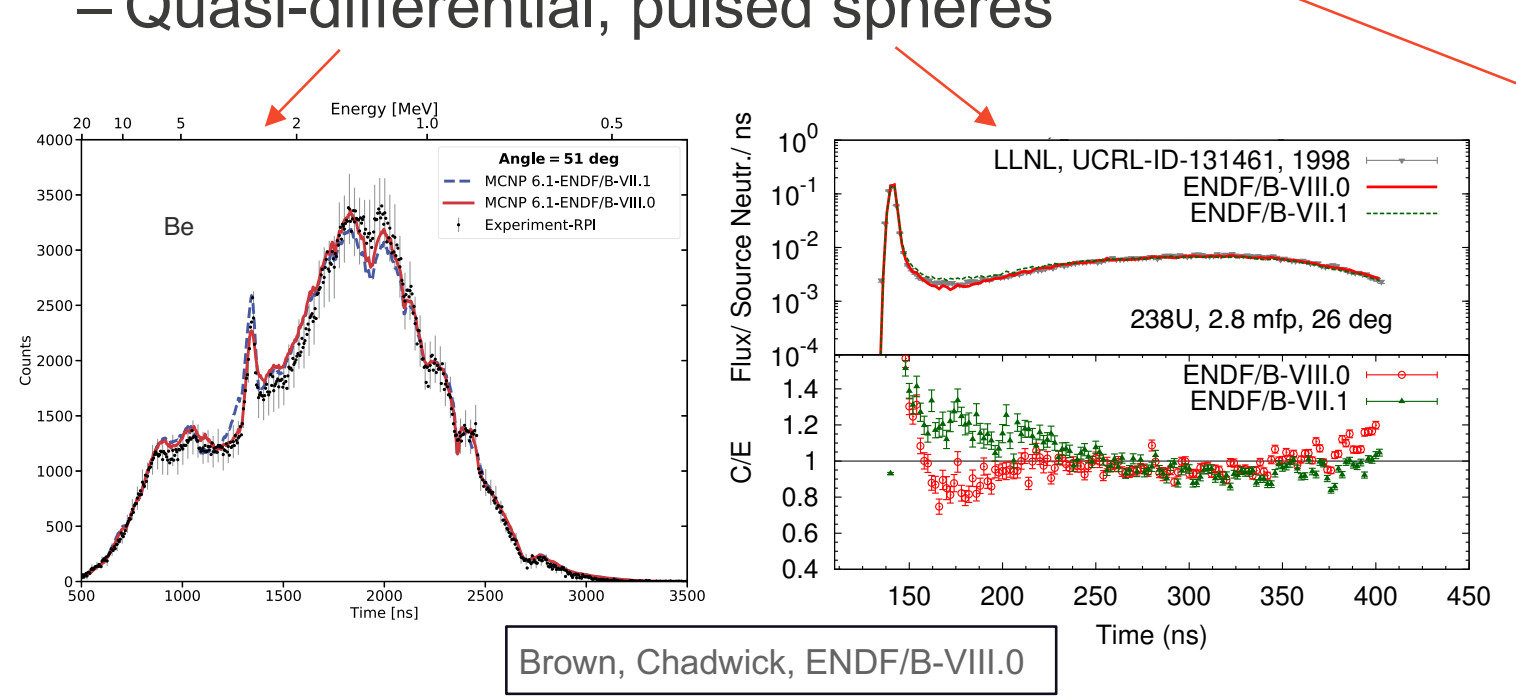

Brown, Chadwick, ENDF/B-VIII.O
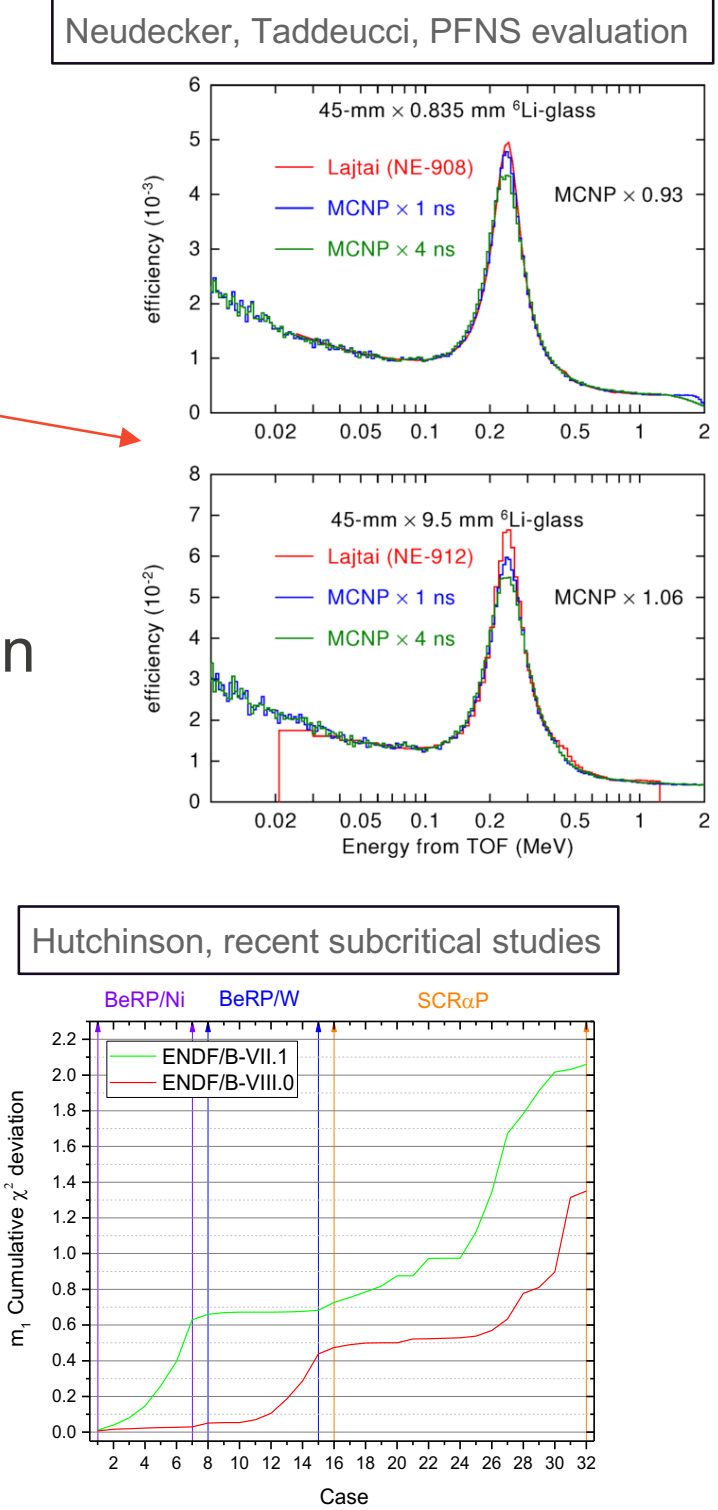


\section{MCNP Development Team}

Team: XCP-3

Terry Adams

Forrest Brown

Colin Josey
Jerawan Armstrong

Jeff Bull

Joel Kulesza
Simon Bolding

Art Forster

Michael Rising

Sriram Swaminarayan (CCS-7)

Computer Support Laura Casswell (WRS-SNA)

Data Team: XCP-5 Jeremy Conlin Nathan Gibson

Wim Haeck

Kent Parsons

University R\&D

William Martin

Christopher Perfetti
Brian Kiedrowski

Todd Palmer 


\section{Future of MCNP}




\section{MCNP6 Now and into the Future}

- Since the time of the MCNP5 and MCNPX merger ( 2013), complexity of the code makes it challenging to maintain

MCNP5 $\sim 100,000$ lines of code $\rightarrow$ MCNP6 $\sim 500,000$ lines of code

- Large amount of code developer time and effort required for:

- Modest improvements, bug fixes, routine maintenance, documentation

- Testing for reliability and robustness - V\&V

- User experiences and new feature developments are beginning to suffer as a result

- Older technology becoming more difficult to maintain, i.e. built-in MCNP plotter

- Challenging to develop and integrate new features

-Ability to run on future architectures is important for future success

- The long-term sustainability and ability to continue to meet mission needs is the primary goal of MCNP6 modernization 


\section{The Vision of the Future Modernized MCNP is Modular}

- Modernization via modularization

- Modular components

- Improved testing $\rightarrow$ provable correctness of the code

- Easier maintainability

- Cleaner code

- Component reuse

- New features more efficiently developed and integrated

- Early career staff excited to work on a more modern code

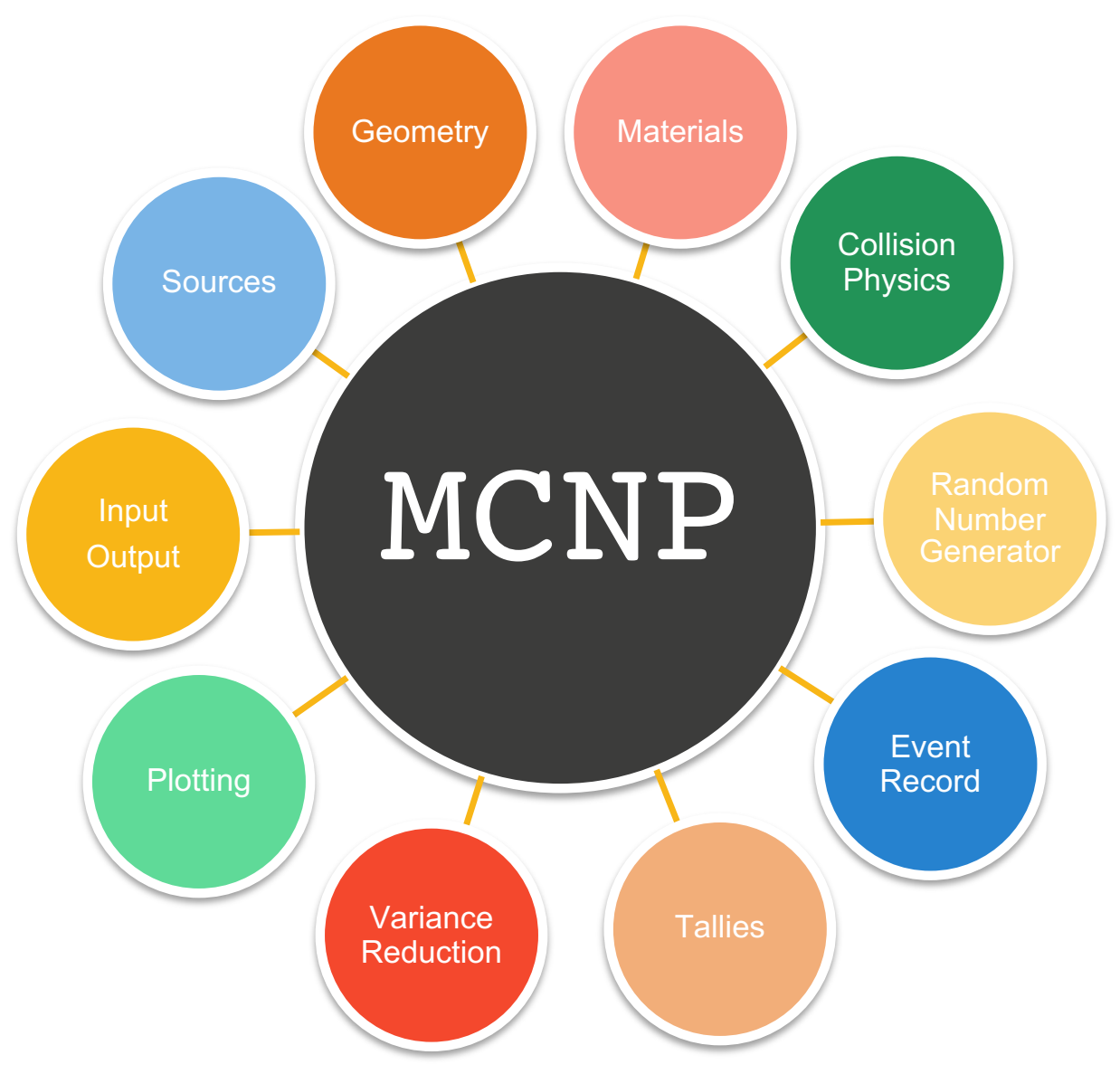




\section{Significant Changes Are Guiding the Future of MCNP}

- While the roots of MCNP go back a long time, there are many changes taking place now looking toward the future

- Adopting modern software development tools

\section{\$ git $\Delta$ cmake Jenkins $\triangle$ atLassian HF}

- Converted to modern direct-access binary file formats, HDF5 - Improved user workflow, e.g. better visualization tools - Faster development within a multi-physics framework

- Reorganization of source routines into modular components enabling future modernization work where needed

- C++ rewrite of several standalone capabilities 


\section{This is an exciting time for MCNP!}

- MCNP code modernization is allowing us to develop new components of the code in modern programming languages using modern tools.

- Exploration of multi-physics applications and how to efficiently and effectively connect to other physics packages is an exciting area of research that MCNP developers are actively involved in.

- Recent and ongoing laboratory directed research and development projects have led to and will lead to more exciting new developments in the MCNP code.

- New unstructured mesh and multigroup cross section tally capabilities

- New sensitivity/perturbation tally methods to be developed in connection with machine learning methods applied to nuclear data improvements and optimized experiment designs 


\section{Summary}

- The history, development and application uses of Monte Carlo particle transport codes at LANL are extensive

- Recent and ongoing investments in MCNP modernization are enabling new code developments using modern programming tools and languages

- New and challenging applications continue to drive new research and developments beyond the rich set of established MCNP capabilities

- The LANL MCNP radiation transport code has been the international gold standard for particle transport applications for over three decades.

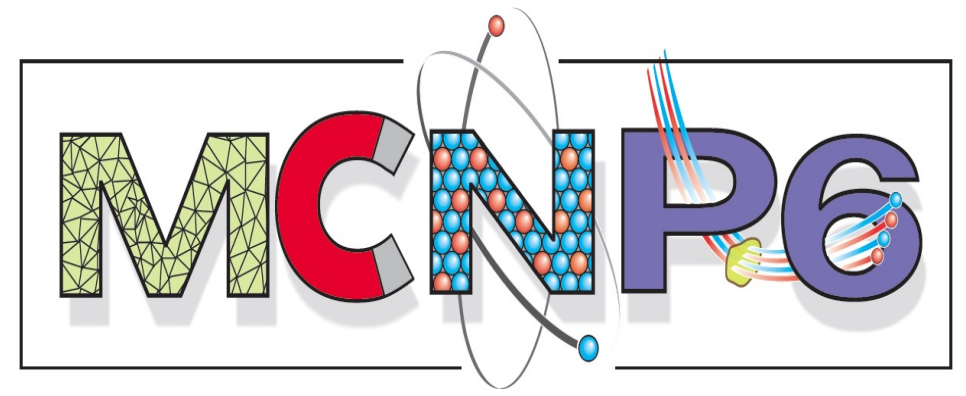




\section{Questions?}

\section{Michael E. Rising, mrising@lanl.gov}

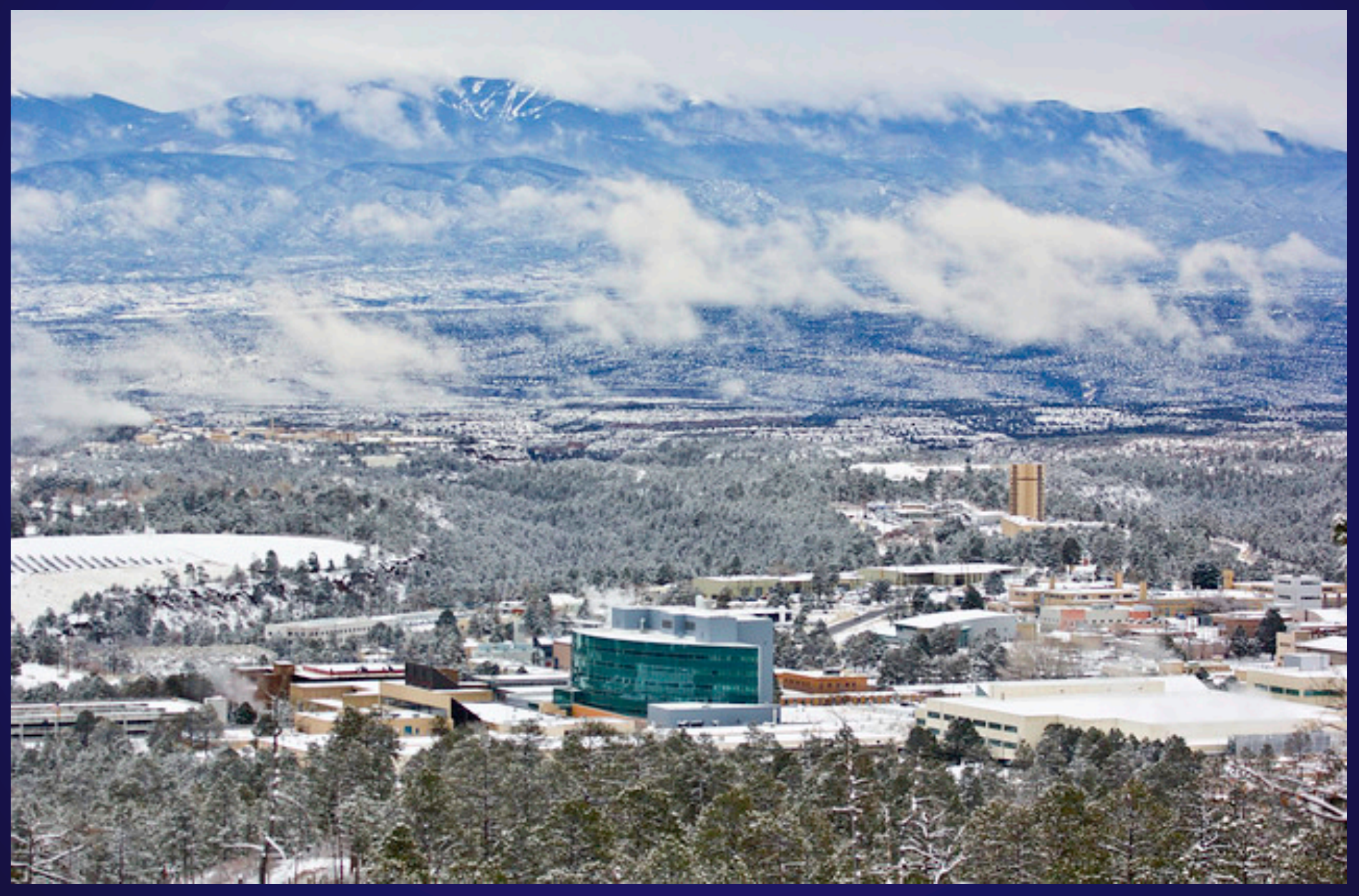




\section{MCNP Modernization}

- Adopted modern software development tools

- Version control system - CVS $\rightarrow$ git

- Build, test and package software - GNU Make $\rightarrow$ CMake \& Ctest

- Repository management / code reviews - TeamForge / Gerrit $\rightarrow$ Bitbucket

- Artifact / issue tracking - TeamForge $\rightarrow$ Jira

- Team communication / wiki - TeamForge $\rightarrow$ Confluence

- Continuous build and testing system - CBTS $\rightarrow$ Jenkins Test Servers

- Testing reporting and monitoring - CBTS $\rightarrow$ Cdash

- Usage and performance reporting - splunk>

- Getting away from "home-grown" tools was not a trivial task but it was essential so developers can spend their time more efficiently'

- Most of these changes are transparent to the user community, but they enable developers to be more responsive to user needs 


\section{MCNP Modernization}

- Conversion to direct-access file capabilities through HDF5 and XDMF

- Complete restart file refactor (runtpe) $\checkmark$

- Particle track output (ptrac) $\checkmark$

- Surface source read/write (SSW/R)

- Standard tally results (mctal)

- Mesh tally results (meshtal) $\checkmark$

- Unstructured mesh elemental edit output (eeout) $\checkmark$

- Others: wwinp, wwone, wwout, srctp, etc.

- Now tools like ParaView, Vislt, etc. are easily included in an engineering workflow with MCNP

- Oak Ridge National Laboratory Pool Critical Assembly - Ref. [J. Kulesza]
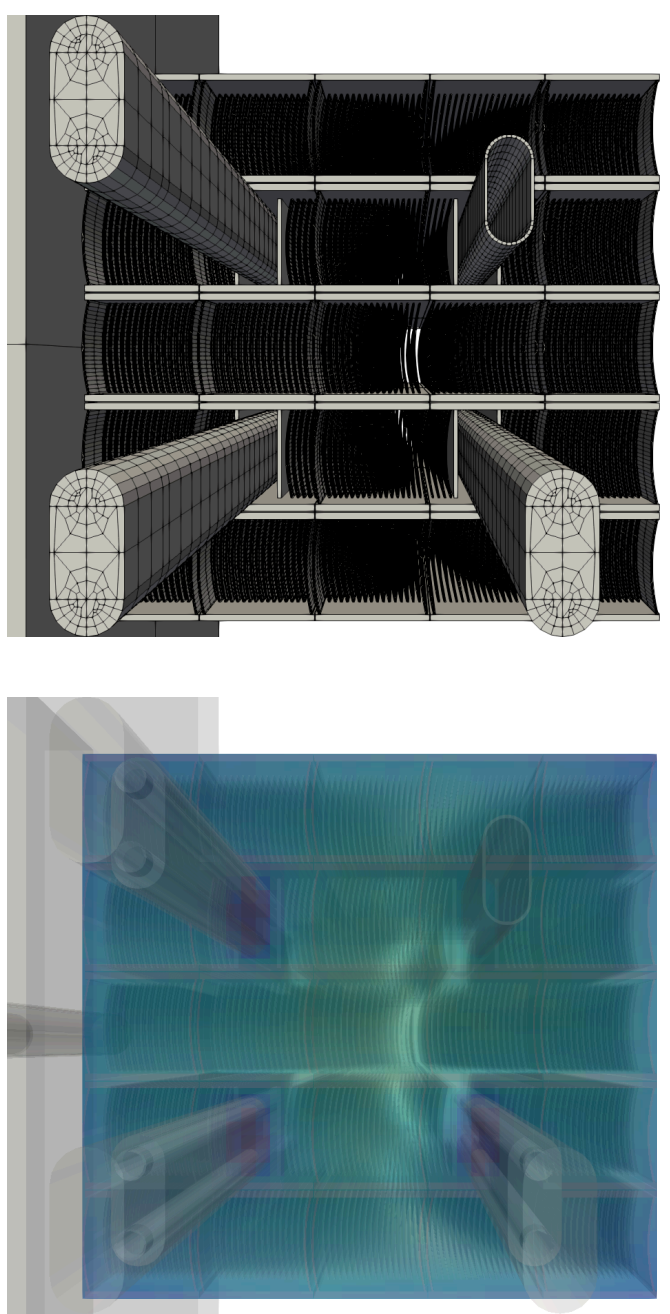


\section{MCNP Modernization}

- Source code modularization, organization, and replacement of unmaintainable source code

- Complete reorganization into components, breaking unnecessary dependencies, is ongoing now

- Modern and robust C++ implementation of general sources (sdef) replaces existing hard-to-maintain and error-prone legacy routines in MCNP

- Modern parallel C++ implementation of event record output (ptrac) with HDF5 file format is complete

- Other improved modular capabilities will continue to be prioritized geometry, tallies, collision physics, variance reduction, etc.

- MCNP6 is fully Fortran 2008 and C++17 standards compliant 


\section{Emerging MCNP Applications for Reactors}

- DOE-NE and other programs use MCNP for reactor design/performance

- Space reactor design/Kilopower

- Design of new types of systems

- Kinetic parameters such as reactivity coefficients

- Shielding

- Microreactors

- Design of new types of systems

- Kinetic parameters such as reactivity coefficients

- Versatile Test Reactor

- Burnup

- Reactivity Coefficients

- Other advanced reactors

- Gas-cooled reactors
Whole Core Pin Power Distribution (w/cc). Step 44
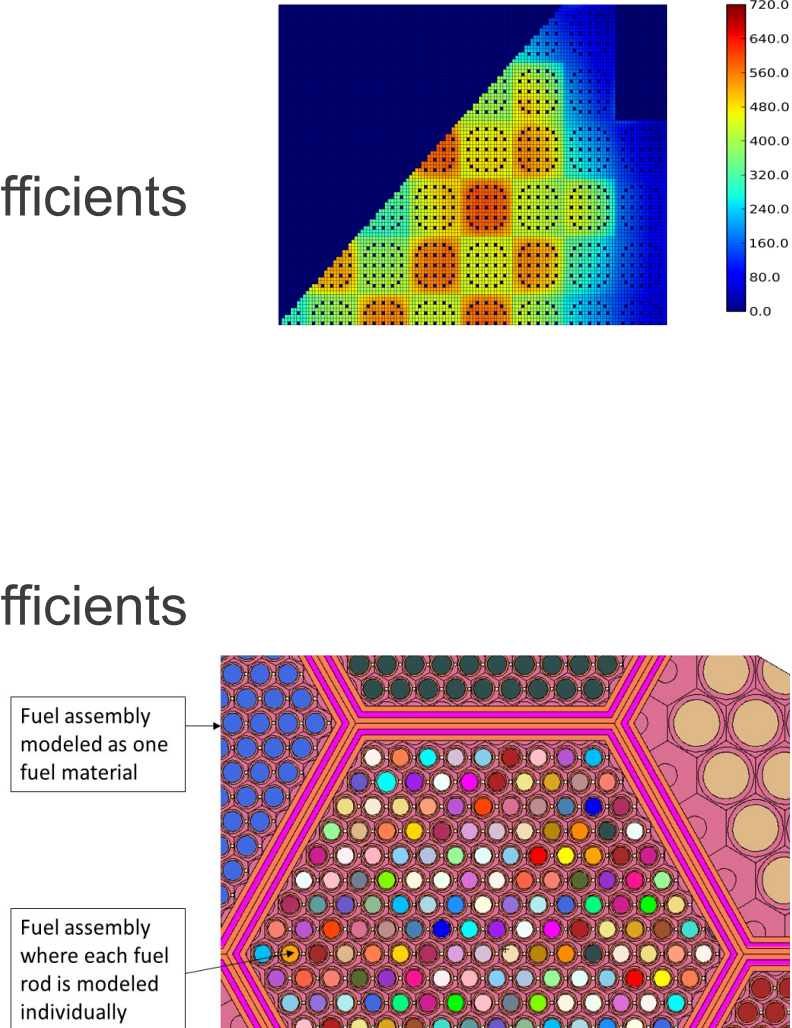

\section{$\rightarrow$}
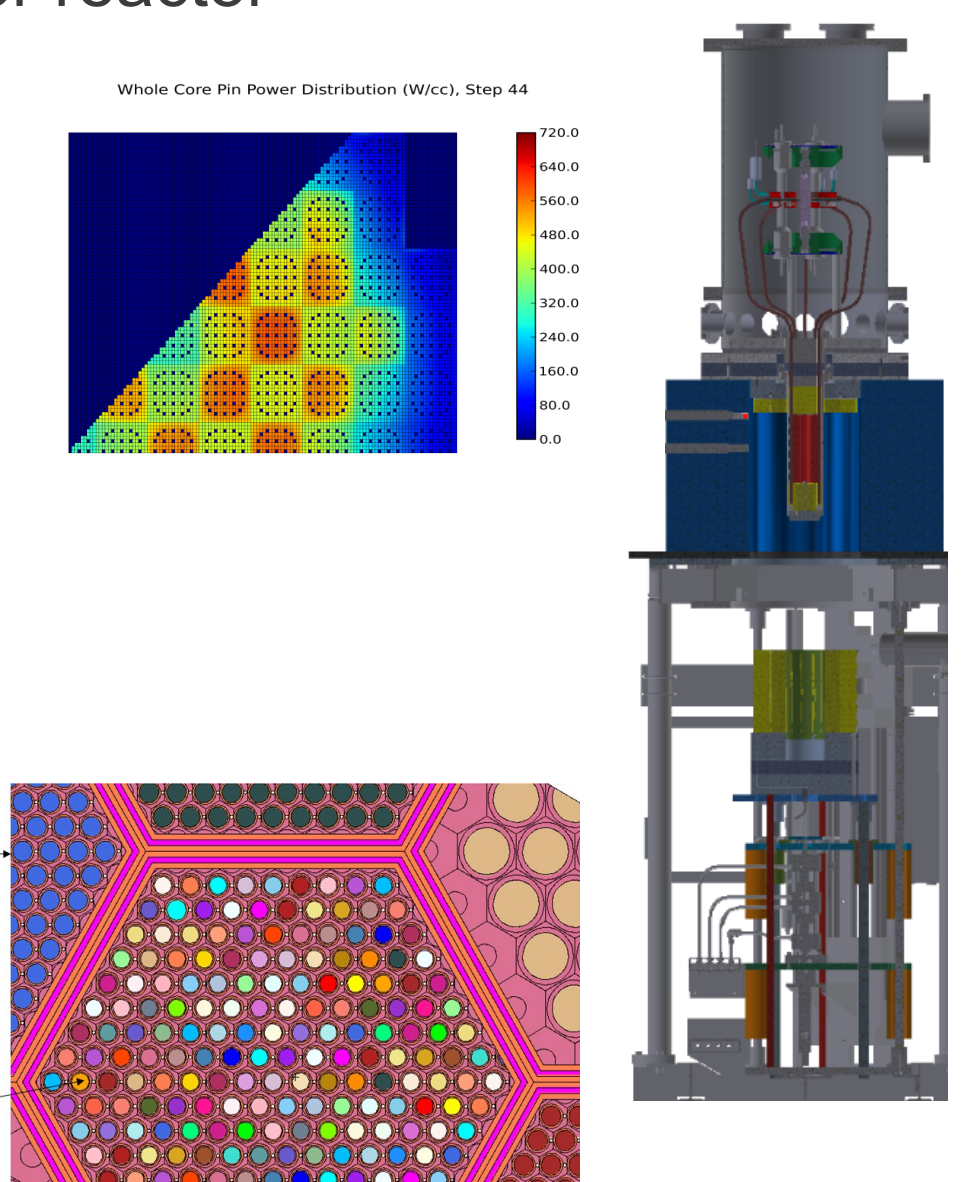


\section{Advanced Monte Carlo Sensitivity/Uncertainty Methods Support R\&D Efforts in Optimization, Validation, and UQ}

- A more direct connection between nuclear data (ND) and applications can be obtained through sensitivity profiles (derivatives) of the application results to the ND observables

- The MCNP6 ${ }^{\circledR}$ code can accurately compute continuousenergy k-eigenvalue (criticality) sensitivity coefficients

- Many new capabilities and areas of applied research are made possible through accurate sensitivity profiles:

- NCSP-funded and established Whisper code

- Benchmark-application similarity assessment

- Calculation of application bias

- Quantification of ND induced uncertainties

- ND assimilation and residual uncertainty assessment

- LDRD ARCHIMEDES experiment optimization project

- Experiment-application similarity assessments and optimization

- LDRD EUCLID project

- Bias assessment to search problematic ND

- ND assimilation for specific applications

- Adding and extending the fixed-source and eigenvalue perturbation methods to all ND
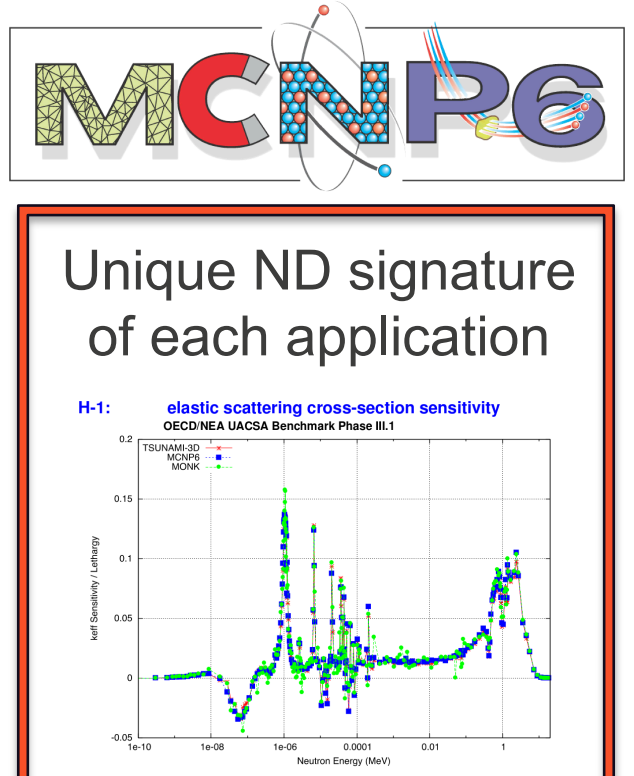

U-238: total cross-section sensitivity
OECDNNEA UACSA Benchmark Phase

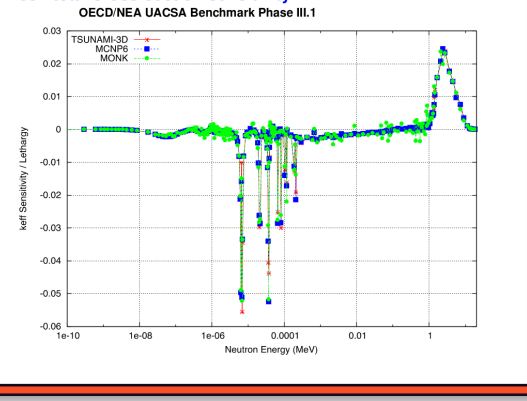

